\title{
2D elastic full-waveform inversion for estimating fluid distribution in hydrocarbon reservoir
}

\author{
Yunosuke IWAKI $^{1}$, Hitoshi MIKADA ${ }^{1}$, Tada-nori GOTO ${ }^{1}$ and Junichi TAKEKAWA ${ }^{1}$ \\ ${ }^{1}$ Dept. of Civil and Earth Res. Eng., Kyoto University
}

\begin{abstract}
Seismic full-waveform inversion (FWI) method has been used to estimate subsurface velocity structure. FWI directly utilizes observed waveforms that could include information on the properties of subsurface materials. In seismic time-lapse surveys, we observe the difference between waveforms as a function of time for the change such as fluid alteration. Residual waveforms between the observed before and after a certain time interval are used to estimate the changes in the fluid distribution in terms of seismic velocities in FWI method. In contrast to the previous FWI applications, our research focuses directly on the properties in the hydrocarbon reservoir in order to estimate the fluid distribution and alteration. We simulate the wave propagation based on the Biot theory that includes the effects of fluid in porous media. The simulation model is composed of a block of sandstone saturated with water and gas. We assume a transition zone around the fluid contact, whose vertical profile of the saturation rate varies gradually in time in this zone. The result inspires that the combination of elastic parameters is necessary for estimating the seismic velocity contact in fluid transition zone relating to the fluid-contact movement by FWI.
\end{abstract}

\section{INTRODUCTION}

Seismic time-lapse survey is conducted for reservoir monitoring to estimate fluid distribution as a function of time. The repeated observation with the same arrangement of source and receivers gives us the seismic waveforms which would change if the fluid distribution in subsurface reservoir changes. The response of seismic waves includes the information of the medium that the seismic waves traveled through. If we can utilize the change in the seismic waveform directly, the fluid alternation would be monitored using seismic exploration methods. The seismic time-lapse experiment has already been carried out by surface seismic survey ${ }^{1)}$.

Considering the effect of the distribution of fluid to the behavior of propagating seismic waves is a key for evaluating the fluid mixture around the fluid contact in the reservoir. Once the relationship between fluid movement and seismic waveform is revealed, we can use the waveform residuals to estimate fluid distribution in subsurface. As an imaging tool using waveform residuals, we present an algorithm based on the 2D elastic full-waveform inversion (FWI) method. FWI method is based on minimizing the misfit function, and updates the model parameter iteratively. This method is applied in many previous papers for solving acoustic problems $^{2)}$, elastic problems ${ }^{3 / 4)}$ and poroelastic problems ${ }^{5}$. In order to consider the fluid variation in hydrocarbon reservoir, we solve a poroelastic problem in a finite-difference scheme. If we could utilize the high resolution nature of FWI, we could be able to see the small change in the subsurface medium in short period of time. It is meaningful to study how the method could be extended to estimate other elastic parameters.

\section{METHOD}

\section{(1) Full-waveform inversion}

FWI is used to estimate the velocity model for acoustic and elastic problems. It seeks the appropriate velocity model based on the minimization of the misfit function S. The misfit function is the difference between observed and calculated waveforms given by

$$
S=\sum_{\text {shots }} \int_{0}^{t} d t \sum_{\text {receivers }}\left[d_{\text {cal }}-d_{o b s}\right]^{2}
$$

We calculate the gradient for model updating to minimize the misfit function $\mathrm{S}$. In this paper, we apply the conjugate gradients method. We need an initial model to start the iterative inversion. In our research, we focus on the seismic time-lapse and use the medium before the fluid alteration as 
already-known model. FWI modifies the initial model to the true model with the waveform residuals decreased.

\section{(2) Biot model}

In order to derive the seismic waveform changed with the fluid alteration, we use the poroelastic model $^{6)}$. The poroelastic model can simulate the wave propagation through the medium consists of solid and fluid phase. In this paper, we use a second-order, finite-difference Biot scheme with staggered grids for computing. The discretized equations are derived from the Biot equations and the velocity-stress formulation ${ }^{7}$. To solve poroelastic equations we need 8 parameters in both fluid and solid phase, but we solve the elastic FWI problem which can handle only 3 model parameters, i.e. P-wave velocity, S-wave velocity and density. We consider the relationship between the fluid distribution and velocity and density parameters distribution to search the fluid movement.

\section{SIMULATION STUDY}

\section{(1) P-wave velocity boundary movement}

Before handling the fluid movement problem, we consider the simple P-wave velocity boundary movement in the elastic problem. Figure 1 shows the simulation model after the boundary movement. We assume the two-layer model, higher P-wave velocity above the boundary and lower below the boundary. The sources are set at $190 \mathrm{~m}$ and $310 \mathrm{~m}$ point at $200 \mathrm{~m}$ in depth. We use the Ricker wavelet with $40 \mathrm{~Hz}$ central frequency as a source function. The 11 receivers are aligned at $200 \mathrm{~m}$ in depth, $200 \mathrm{~m}-300 \mathrm{~m}$ per $10 \mathrm{~m}$. The model size is $500 \mathrm{~m} \mathrm{x}$ $500 \mathrm{~m}$, but the inversion area is $[200 \mathrm{~m}-300 \mathrm{~m}] \mathrm{x}$ [300m- $400 \mathrm{~m}]$, enclosed by the purple line shown in Figure 1.

The P-wave velocity distributions of model-updated area are shown in Figure 2. We assume a $20 \mathrm{~m}$ transition zone around the velocity boundary with a global velocity change. The original velocity boundary is at $380 \mathrm{~m}$ in depth, and is shifted to $320 \mathrm{~m}$ after the movement. Figure 2(a) is the model after the boundary movement, and the true model to be estimated. Figure 2(b) is the distribution before the boundary movement. We regard it as the known-model, and use it for the initial model of FWI simulation. After the 30 iterations by FWI, we obtained the resultant model shown in Figure 2(c). Around the $320 \mathrm{~m}$ in depth, a strong velocity contrast was obtained. Thus, the P-wave velocity movement can be approximately estimated by using the known model before the movement for FWI.

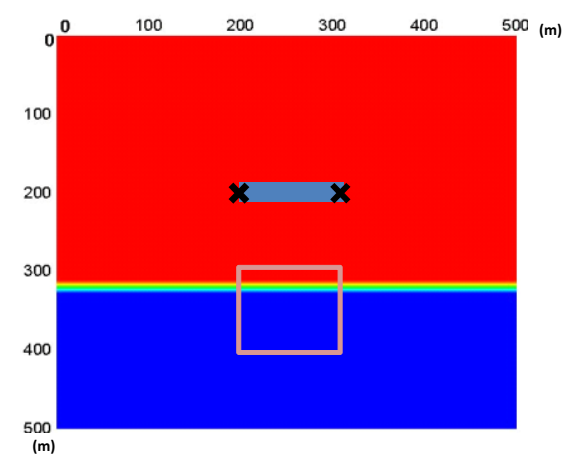

Figure 1 The simulation model for P-wave velocity boundary movement.

a)

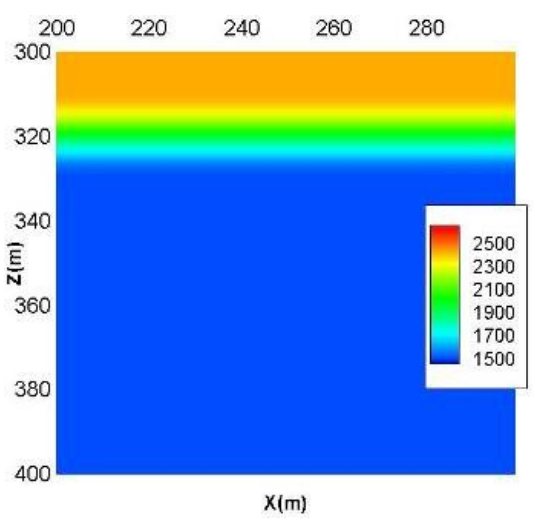

b)

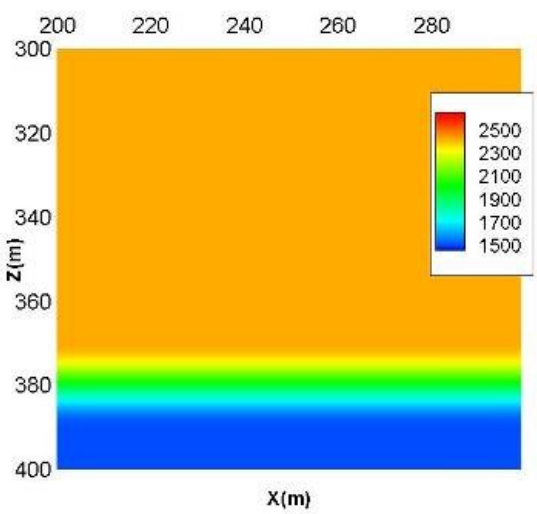

c)

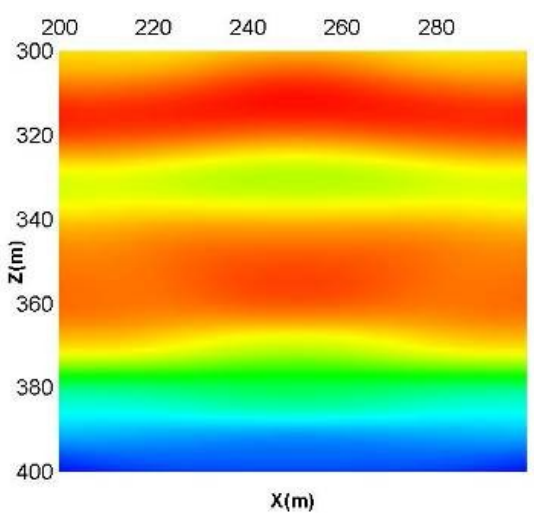

Figure 2 Simulation result for P-wave velocity movement: (a) true model, (b) initial model, (c) FWI result. 
The inversion result, however, shows some difficulties. First, the boundary before the movement remained after the inversion (around $380 \mathrm{~m}$ in depth). Second, the absolute value of $\mathrm{P}$-wave velocity was not estimated. Figure 3 shows the misfit function versus the iteration number. Figure 4 shows the waveform residuals at all receivers when we use the source at $(190 \mathrm{~m}, 200 \mathrm{~m})$. Figure 4(a) is the difference of waveforms between true model and initial model and $\mathbf{4 ( b )}$ is one between true model and FWI result. The reflected wave difference decreased for P-P wave (around $0.15 \mathrm{~s}$ and $0.20 \mathrm{~s}$ ). The misfit function converged about $30 \%$ and the waveforms still have the differences.

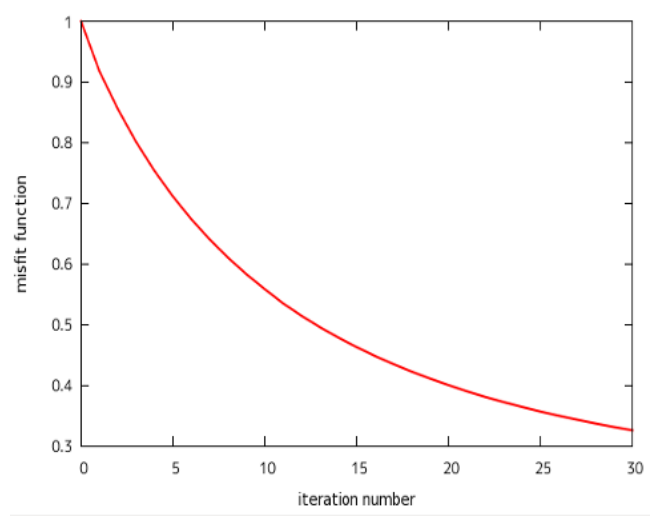

Figure 3 Misfit function for P-wave velocity change.

a)

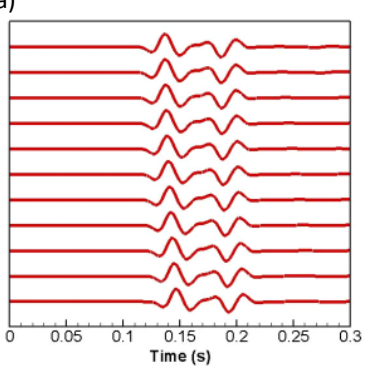

b)

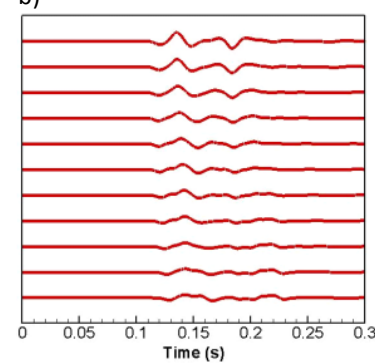

Figure 4 Wave residuals between: (a) initial and true model, (b) FWI result and true model.

\section{(2) Fluid boundary movement}

We apply our FWI method for the estimation of the fluid movement. Figure 5 shows the whole model after the fluid boundary movement. The media consists of sandstone in solid phase. In fluid phase, the upper layer is saturated by gas and the lower is saturated by water. The sources are set at $60 \mathrm{~m}$ and $120 \mathrm{~m}$ point at $60 \mathrm{~m}$ in depth. Same as the case in P-wave velocity boundary movement, we use the Ricker wavelet with $40 \mathrm{~Hz}$ central frequency as a source function. The 16 receivers are aligned at
$60 \mathrm{~m}$ in depth, $60 \mathrm{~m}-120 \mathrm{~m}$ per $4 \mathrm{~m}$. The model size is $300 \mathrm{~m} \times 180 \mathrm{~m}$, but the inversion area is $[60 \mathrm{~m}-120 \mathrm{~m}]$ $\mathrm{x}[60 \mathrm{~m}-240 \mathrm{~m}]$, enclosed by the purple line. In fluid contact, we apply a transition zone. The water ratio increases gradually along with the depth. In this simulation model, we assume the $20 \mathrm{~m}$ transition zone originally around $180 \mathrm{~m}$ in depth, $120 \mathrm{~m}$ after the fluid movement. In the transition zone, the fluid is treated as fluid mixture. The bulk modulus is derived by Reuss average and the density is derived by Voigt average.

By the fluid contact movement, the distribution of all elastic parameters, $\mathrm{P}$-wave velocity, S-wave velocity and density, would be changes. In this paper, we apply the FWI to estimate P-wave velocity distribution. In the transition zone, P-wave velocity saturated by water and gas is near the value of the media saturated by gas only, so the P-wave velocity boundary is seen at the bottom of the transition zone. The observed data for true model is derived by the wave propagation calculation based on Biot equation. The wave propagation in the inversion process is calculated as the elastic problem. The P-wave velocity model of the target area is shown in Figure 6. Each (a), (b) and (c) in Figure 6 corresponds the true model, initial model and one obtained by FWI, respectively. After the 30 iterations, we can see the strong velocity contrast at $130 \mathrm{~m}$ in depth which fits to the true model.

Such as in the case of variation of P-wave velocity boundary, the contact before the movement remained and the absolute P-wave velocity was not derived. The misfit function shown in Figure 7 converged to $20 \%$. This is because the S-wave velocity and density have different value between true model and the inversion result. Figure 8 shows the waveform residuals at all receivers when we used the source at $(60 \mathrm{~m}, 60 \mathrm{~m})$. Figs. 8(a) and (b) are the waveform differences between true model and initial model, and between true and one obtained by FWI, respectively. The reflected wave difference decreased for P-P wave (around $0.14 \mathrm{~s}$ and $0.21 \mathrm{~s}$ ). In the assumption we used for the fluid mixture, the $\mathrm{P}$-wave velocity drastically changes at the bottom of the transition zone. To estimate the state of transition zone, the S-wave velocity or density distribution should be considered.

\section{CONCLUSION}

We demonstrated the estimation of contact movement by elastic FWI algorithm in time-lapse case. First, we estimated a simple velocity boundary movement in elastic problem. Second, we apply our FWI method to fluid movement for media composed of fluid and solid phases. The P-wave 
velocity contact can be estimated as a separate contact. The parameter estimation in transition zone requires some process to the observed waveform to get close to the true image. The source with higher frequency ranges will help to estimate further fluid distribution in the transition zone. We should consider the S-wave velocity and density inversion to improve the inversion result.

\section{REFERENCES}

1) Kasahara, J., Ito, S., Hasada, Y., Fujii, N., Ikuta, R., Tsuruga, K., Fujiwara, T., Yamaoka, K., Nishigami, K. and Ito, K., 2012, Time lapse experiment using the seismic ACROSS source near the Nojima-fault in Awaji Island-Imaging and travel-time changes by injection and rain-fall effects, Proceedings of the $126^{\text {th }}$ SEGJ conference, 106-109 (in Japanese with English abstract).

2) Tarantola, A., 1984, Inversion of seismic reflection data in the acoustic approximation, Geophysics, 49, No.8, 1259-1266.

3) Tarantola, A., 1988, Theoretical background for the inversion of seismic waveforms, including elasticity and attenuation, Pure and Applied Geophysics, 128, Nos. 1/2, 365-399.

4) Sears, T. J., Barton, P. J. and Singh, S. C., 2010, Elastic full waveform inversion of multicomponent ocean-bottom cable seismic data: Application to Alba Field, U. K. North Sea, Geophysics, 75, No.6, R109-119.

5) De Barros, L., Dietrich, M. and Valette, B., 2010, Full waveform inversion of seismic waves reflected in a stratified porous medium, Geophysics Journal International, 182, 1543-1556.

6) Biot, M.A., 1956, Theory of propagation of elastic waves in a fluid-saturated porous solid, I- Low-frequency range, Journal of the Acoustic Society of America, 28, 168-178.

7) Wenzlau, F., Müller, T.M., 2009, Finite-difference modeling of wave propagation and diffusion in poroelastic media, Geophysics, 74, No.4, T55-T66.

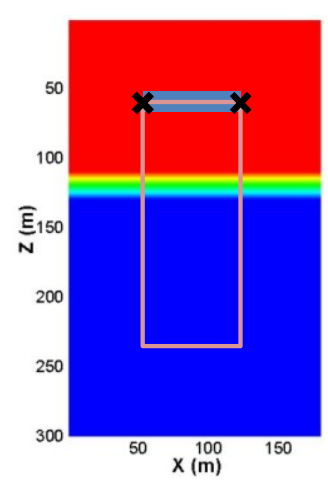

Figure 5 The simulation model for fluid boundary movement.
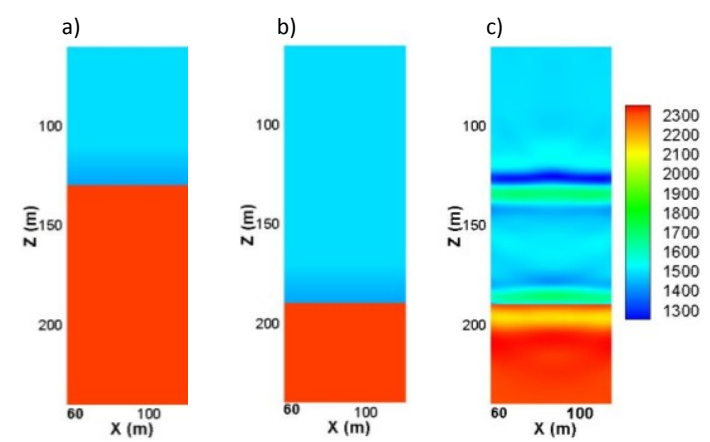

Figure 6 Simulation result of $\mathrm{P}$-wave velocity distribution: (a) true model, (b) initial model, (c) FWI result.

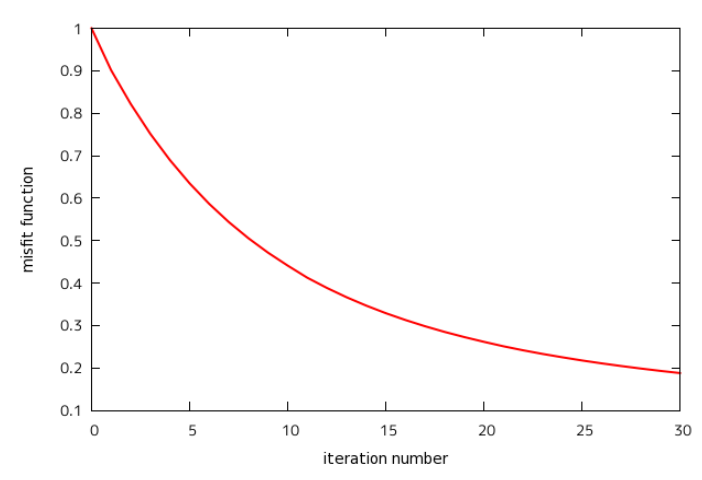

Figure 7 Misfit function for P-wave velocity change.

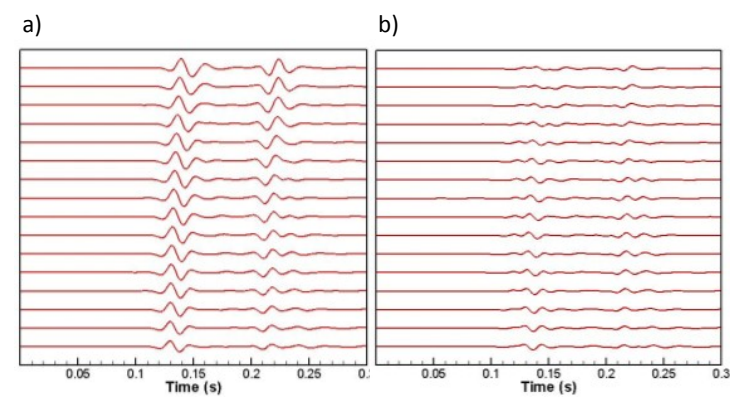

Figure 8 Wave residuals between: (a) initial and true model, (b) FWI result and true model. 